\section{Assessment of Cognitive Impairment in Patients with Cerebral Infarction by MMSE and MoCA Scales}

\section{Sir,}

Cognitive impairment is a common complication of cerebral infarction. Early diagnosis and intervention will be beneficial to the rehabilitation of neurological function in patients with cerebral infarction. A study has shown that cognitive rehabilitation training after early diagnosis of cerebral infarction patients with cognitive impairment can prevent or delay the development of dementia. ${ }^{1} \mathrm{At}$ present, there are no clear diagnostic criteria for cognitive impairment after cerebral infarction.

Mini mental state exam (MMSE) is an important method for screening and evaluating cognitive impairment. With the deepening of research, many researchers believe that MMSE can only detect patients with severe cognitive impairment and dementia, it lacks sensitivity and specificity to mild cognitive impairment $(\mathrm{MCl})$, and cannot effectively identify $\mathrm{MCl}$, normal cognitive aging and early Alzheimer's disease. ${ }^{2}$ Montreal cognitive assessment (MoCA) scale was developed by referring to the setting of cognitive items and scoring criteria of MMSE and combining a large number of clinical experiences. Studies have shown that MoCA can be used to screen for mild cognitive impairment in patients with chronic kidney disease before dialysis. ${ }^{3}$

The aim of this study was to explore the application value of MMSE and MoCA scales in assessing cognitive impairment in patients with cerebral infarction.

One hundred and thirty-one patients with single lesion confirmed by CT or MRI, who have had cerebral infarction for three months, were selected as the study subjects. Patients with severe hearing impairment, language impairment, visual impairment, moderate to severe depression and mental disease, cognitive impairment or dementia before diagnosed, the age over 80 years and the education level below 5 years, were excluded. The subjects were respectively tested by two standardised trained testers with the MMSE and MoCA scales in quiet rooms. The test interval between MMSE and MoCA was more than one day, and all tests were completed at one time. MMSE included tests on orientation, memory, computation, language, visual space, use and attention, with a total score of 30 points. MoCA included tests on visual space and execution, naming, attention, language, abstraction, delayed memory, time and location orientation, with a total score of 30 points. When the scores of MMSE and MoCA were respectively lower than 24 and 26 , the patients would be judged as cognitive impairment. SPSS version 25 software was used to analyse the data, and paired t-test and Chi-square test were carried out. $\mathrm{P}<0.05$ was statistically significant.

Among 131 patients, 74 were males (56.49\%) and 57 were females $(43.51 \%)$. Age ranged from 41 to 72 $(64.51 \pm 2.07)$ years. Education level was from 6 to 11 $(9.54 \pm 0.73)$ years. The location of infarct was frontal lobe in 31 cases $(23.66 \%)$, temporal lobe in 38 cases $(29.01 \%)$, basal nucleus in 32 cases $(24.43 \%)$, and cerebral ganglion in 30 cases $(22.90 \%)$.

The MMSE of 131 patients were 16-29 (25.74 \pm 2.92$)$ and the MoCA were 5-29 (20.15 \pm 4.78$)$. MoCA scores were lower than MMSE scores, and the difference had statistical significance $(p<0.001)$. Fifty-four cases $(41.22 \%)$ were diagnosed as cognitive impairment by MMSE. One hundred and twenty cases $(91.60 \%)$ were diagnosed as cognitive impairment by MoCA. The proportion of cognitive impairment diagnosed by MoCA was significantly higher than that by MMSE $(p<0.001)$.

Some studies shown the items of MMSE scale were relatively rough and lack of evaluation items such as visual spatial organisations and abstract thinking, so it was easy to miss the diagnosis of mild cognitive impairment. 4 The results of this study showed that MoCA was more likely to reflect cognitive decline after cerebral infarction than MMSE. This conclusion was consistent with the conclusion reported by Smith et al. that the sensitivity of MoCA to recognise mild cognitive impairment was significantly higher than that of MMSE. 5 It suggested that MoCA was more suitable for early diagnosis of cognitive impairment after cerebral infarction. The reason may be that the MMSE and MoCA scales focus on different cognitive domains. MMSE is designed to screen for Alzheimer's disease. It has more items to detect memory and cortical function, but fewer items to reflect subcortical executive function. MoCA, on the contrary, has more projects to detect execution functions. The cognitive impairment of cerebral infarction is mainly executive impairment, and the impairment of memory and cortical function is mild. Therefore, the sensitivity of MoCA in screening cognitive impairment in patients with cerebral infarction is high, while the sensitivity of MMSE to executive impairment is low. It should be noted that MoCA scores are influenced by factors such as gender, age and educational level. Clinical application of MoCA sale should be assessed further according to individual differences of patients. 


\section{ACKNOWLEDGEMENT:}

This study is supported by the Clinical Special Science Foundation of Science and Technology Department of Jiangsu Province, China (No. BL2014028).

\section{CONFLICT OF INTEREST:}

Authors declared no conflict of interest.

\section{AUTHORS' CONTRIBUTION:}

$\mathrm{CO}$ : Analyzed data and organized the manuscript. $X L$, JG, KX: Collected information of patients.

$\mathrm{CL}, \mathrm{XA}$; Conducted the project, provided study ideas and kept the research running smoothly.

\section{REFERENCES}

1. Kuznetsova EB, Salina EA, Sholomov II. The possibility of treatment of cognitive impairment in the complex therapy of patients with the consequences of cerebral infarction. Zh Nevrol Psikhiatr Im S S Korsakova 2015; 115:103-6.

2. Mamikonyan E, Moberg PJ, Siderowf A, Duda JE, Have TT, Hurtig $\mathrm{HI}$, et al. Mild cognitive impairment is common in Parkinson's disease patients with normal Mini-Mental State Examination (MMSE) scores. Parkinsonism Relat Disord 2009; 15:226-31.
3. Paraizo Mde A, Almeida AL, Pires LA, Abrita RS, Crivellari MH, Pereira Bdos S, et al. Montreal Cognitive Assessment (MoCA) screening mild cognitive impairment in patients with chronic kidney disease (CKD) pre-dialysis. J Bras Nefrol 2016; 38:31-41.

4. Nazem S, Siderowf AD, Duda JE, Have TT, Colcher A, Horn SS, et al. Montreal cognitive assessment performance in patients with parkinson's disease with "normal" global cognition according to mini-mental state examination score. J Am Geriatr Soc 2009; 57:304-8.

5. Smith T, Gildeh N, Holmes C. The Montreal cognitive assessment: Validity and utility in a memory clinic setting. Can $J$ Psychiatry 2007; 52:329-32.

Chunying Ou, Chuanling Li, Xiaolei An, Xiaobin Li, Jing Guo and $\mathrm{Ke} \mathrm{Xu}$

Department of Neurology, Xuzhou Central Hospital, Jiangsu Province, 221000, China

Correspondence to: Dr. Chuanling Li, Department of Neurology, Xuzhou Central Hospital, Jiangsu Province, 221000, China E-mail:licl318@126.com

Received: December 24, 2018; Revised: May 26, 2019 Accepted: July 04, 2019 